\title{
Advanced computational technique based on kriging and Polynomial Chaos Expansion for structural stability of mechanical systems with uncertainties
}

\author{
E. Denimal · J.-J. Sinou $(\mathbb{D}$
}

Received: 9 March 2021 / Accepted: 10 July 2021 / Published online: 9 September 2021

(C) The Author(s) 2021

\begin{abstract}
In this paper, a numerical strategy based on the combination of the kriging approach and the Polynomial Chaos Expansion (PCE) is proposed for the prediction of buckling loads due to random geometric imperfections and fluctuations in material properties of a mechanical system. The original computational approach is applied on a beam simply supported at both ends by rigid supports and by one punctual spring whose location and stiffness vary. The beam is subjected to a deterministic axial compression load. The PCE-kriging meta-modelling approach is employed to efficiently perform a parametric analysis with random geometrical and material properties. The approach proved to be computationally efficient in terms of number of model evaluations and in terms of computational time to predict accurately the buckling loads of a beam system. It is demonstrated that the buckling loads are substantially impacted not only by both the location and the stiffness of the spring, but also by the random parameters.
\end{abstract}

Keywords Buckling $\cdot$ Kriging $\cdot$ Polynomial Chaos Expansion · Rayleigh-Ritz $\cdot$ Structural stability $\cdot$ Uncertainties

\section{Introduction}

The problem of structural stability of mechanical systems [1], such as buckling of beam structures under axial compressive loading has attracted the attention of many researchers. Generally speaking, the first step in engineering for the prediction of buckling is to consider idealized structures with mathematically exact geometries. In reality, structures have imperfections due to the manufacturing process and cannot be considered as homogeneous or geometrically exact. So, the effect of material and geometric imperfections needs to be considered for a robust prediction of buckling and for the estimation of the main differences with the idealized buckling problem. Also, many researches carried out in the field of structural stability theory and practice for buckling of beams were focused

E. Denimal

Univ. Gustave Eiffel, Inria, COSYS/SII, I4S, Campus de Beaulieu, 35042 Rennes, France

e-mail: enora.denimal@inria.fr

J.-J. Sinou

Laboratoire de Tribologie et Dynamique des Systèmes, UMR CNRS 5513, École Centrale de Lyon, 36 avenue Guy de Collongue, 69134 Écully Cedex, France

J.-J. Sinou $(\bowtie)$

Institut Universitaire de France, 75005 Paris, France

e-mail: jean-jacques.sinou@ec-lyon.fr 
on the effects of various sources of imperfections. The issue of initial imperfections (i.e. initial displacement that exists in the unloaded case) in beam impact buckling problems was one of the first questions addressed [2-9]. Many researchers have also demonstrated the significant impact of other types of imperfections such as geometric imperfections, fluctuations in material properties, non-perfect boundary conditions and misalignment in the loading [10-14]. Nowadays, sophisticated numerical methods exist, capable of integrating several sources of uncertainties and imperfections in the structural stability analysis when analyses based on analytical developments are no longer practicable. However, many approaches based on Monte Carlo Simulation (MCS) or approximate methods may require prohibitive computation times or become unusable if a large number of uncertain parameters are taken into account. One of the major challenges in the assessment of buckling of mechanical structures is therefore to propose efficient and robust numerical techniques in order to estimate not only the effects of the physical properties described by means of deterministic parameters but also to quantify the dispersions on the results by considering imperfections, thanks to several random variables.

When studying engineering structures, one usually faces many different types of uncertainties. On the one hand, parametric studies on a few parameters are often considered to observe their influence on some outputs that describe the system behaviour and/or to make choices in the design stage $[15,16]$. These parameters usually evolve in a given interval chosen by the engineer. When the numerical cost related to the model evaluation or when many parameters are considered, it might be numerically too expensive to perform comprehensive parametric studies. One must then take decisions based on a few evaluations of the model and predict its behaviour for other values of the parameters. This uncertainty, related to the lack of evaluated points, is called epistemic and can be reduced by considering more evaluations of the model (and so increase the numerical cost). Surface response approaches are well adapted for these types of issues, the most well known is the polynomial regression. If the latter is easy to understand and implement, it has strong limitations when non-linear behaviours and/or when functions with discontinuities or strong variations must be approximated. Other approaches are then more appropriate as kriging $[15,16]$ or Support-Vector Regression (SVR) [17]. On the other hand, many uncertainties are often present due to environmental changing, manufacturing tolerances, etc. Different modelling are possible for these uncertainties, as random variables with probability density function (PDF), fuzzy variables, etc. This uncertainty is usually referred as aleatory as it cannot be reduced and adding more points would only lead to a better knowledge of the PDF for example. Using random variables remains one of the most traditional and used way to model them. Depending on the modelling of the uncertainty and of the output information required, different approaches exist such as Monte Carlo Simulations (MCS) [18], Polynomial Chaos Expansion (PCE) [19,20], perturbation method [21], fuzzy sets [22], probability transformation method $[23,24]$, etc. Considering the variation of these parameters can lead to important variations of the structural properties, and it is then necessary and essential to do wise design choices by considering these uncertainties to ensure the efficiency and robustness of the structure. As structures are often subjected to both uncertainties, namely epistemic and aleatory, approaches able to deal with both of them are of great interest.

In this framework, in [25], Li et al. proposed to deal with both epistemic and aleatory uncertainty by coupling evidence theory and statistical analysis to compute the lower and upper bounds of a probability of failure. In [26], Wu et al. proposed the Unified Interval Stochastic Sampling (UISS) approach to deal with both types of uncertainties. An initial sampling related to the random parameters is created, and an interval analysis is performed on each of them to propagate the epistemic uncertainty. They finally compute the lower and upper bounds of the structural buckling loads for different structures. Finally, in [27], Feng et al. coupled random parameters and interval analysis for linear static analysis. If these approaches deal with the problem of mixed uncertainty, their cost remain in some cases high requiring numerous model evaluations. Moreover, the formulations are aimed to finally obtain upper and lower bound of the PDF of a quantity of interest. However, in many engineering applications, one wants the evolutions of stochastic physical parameters for large parametric studies. In [28], Denimal et al. proposed a hybrid meta-model that mixed epistemic and aleatory uncertainties by coupling PCE and kriging. The proposed formulation permits to consider large parametric studies when random parameters are present and avoid the prohibitive Monte Carlo/scanning-based methods traditionally used. Such a formulation presents also a major advantage, which is the direct access to the Sobol indices through PCE formulation. This facilitates the realization of sensitivity analysis 
to get deep insights in the role played by different parameters, which is not possible with the different approaches presented previously.

The objective of the present work is then to predict the buckling behaviour of a mechanical system when the two kinds of uncertainties are present, namely epistemic and aleatory. The considered system is a beam where all geometrical and material properties are considered as uncertain and can vary. This uncertainty is modelled through a PDF for each parameter. The buckling properties of the beam system are studied when a spring is added to the latter. The equation of motion of the system is obtained with the Rayleigh-Ritz method. The spring location and stiffness can vary, modifying strongly the buckling properties. Indeed, the buckling loads as well as the buckling mode shapes are impacted by this additional stiffness. Maximum buckling loads are observed at specific location of the spring and a saturation effect is observable when the spring stiffness increases. By considering the uncertainties related to the beam model, the stochastic properties of the buckling loads are analysed by using the PCE-kriging meta-model. It is demonstrated that with a low number of model evaluations and a drastic reduction of the computational time, the meta-model is able to predict accurately the different buckling loads and their stochastic moments. Finally, a sensitivity analysis based on the Sobol indices directly assessed from the PCE-kriging formulation is achieved to investigate in depth the influence of the different beam model parameters.

\section{Hybrid surrogate model presentation}

In the present work, random variables are employed to describe the aleatory uncertainty as it is one of the most traditional and used way to model it. The epistemic uncertainty is modelled through parametric variables that can take the values in a given interval. They will be denoted as parametric and random uncertainties or variables in the following for clarity considerations. Each of them is treated in a different manner to take into account their characteristics.

The general problem consists then in approximating the function $f$ that depends on $\mathbf{x}$ and on $\boldsymbol{\xi}$, where $\mathbf{x}$ is the vector of parametric variables of dimension $n$ and $\xi$ the vector of random variables of dimension $m$.

\subsection{Kriging}

For epistemic uncertainties, surface response approaches are well adapted due to their non-intrusive characteristics. The most well-known one being the polynomial regression, despite its limited efficiency especially when nonlinear behaviours or when functions with discontinuities are approximated. Another approach is kriging, which is the best unbiased predictor [16]. It has proven its efficiency in many applications, even for non-linearities and/or discontinuities [15], and will be used in the following.

Let $h$ denote the function to be approximated from a limited number of evaluations that depends on $\mathbf{x} \in \mathbb{R}^{n}$, then a kriging approximation of $h$ is given by [16,29]

$y=h(\mathbf{x}) \approx \widehat{h}(\mathbf{x})=\sum_{k=0}^{q} g_{k}(\mathbf{x}) \beta_{k}+\mathcal{Z}(\mathbf{x})$,

where $\left(g_{k}\right)$ is a basis of $q$ orthogonal regression functions, often chosen as polynomials of low order (up to order 2), $\boldsymbol{\beta}$ the vector of the regression coefficients to be determined and $\mathcal{Z}$ a zero-mean Gaussian process of variance $\sigma^{2}$. The covariance matrix is given by

$\mathbb{E}\left[\mathcal{Z}(\mathbf{x}), \mathcal{Z}\left(\mathbf{x}^{\prime}\right)\right]=\sigma^{2} \mathcal{R}\left(\mathbf{x}, \mathbf{x}^{\prime}\right)$,

where $\mathcal{R} \in[0,1]$ is the spatial correlation function and $\mathbb{E}$ the expectation operator. The correlation function is usually unknown but constructed from a family of kernel functions parameterized by the hyper-parameter $\boldsymbol{\theta}$, and depends on the distance between $\mathbf{x}$ and $\mathbf{x}^{\prime}$. If $n>1$, then the correlation function is constructed by taking the tensor 
Table 1 Examples of correlation function family in dimension 1

\begin{tabular}{ll}
\hline Kernel & $k(\theta, d)$ \\
\hline Gaussian & $\exp \left(-\frac{d^{2}}{2 \theta^{2}}\right)$ \\
Exponential & $\exp \left(-\frac{|d|}{\theta}\right)$ \\
Matérn 5/2 & $\left(1+\frac{\sqrt{5}|d|}{\theta}+\frac{5 d^{2}}{3 \theta^{2}}\right) \exp \left(-\frac{\sqrt{5}|d|}{\theta}\right)$ \\
Matérn 3/2 & $\left(1+\frac{\sqrt{3}|d|}{\theta}\right) \exp \left(-\frac{\sqrt{3}|d|}{\theta}\right)$
\end{tabular}

product of a univariate kernel function family $k$ :

$\mathcal{R}\left(\boldsymbol{\theta}, \mathbf{x}, \mathbf{x}^{\prime}\right)=\prod_{j=1}^{n} k\left(\theta_{j}, d_{j}\right)$,

where $\theta_{j}$ is the value of $\boldsymbol{\theta}$ in the $j$-th dimension, and $d_{j}=x_{j}-x_{j}^{\prime}$ the distance between $\mathbf{x}$ and $\mathbf{x}^{\prime}$ in the $j$-th dimension. For isotropic kriging, $\theta_{j}=\theta$ is a constant, and in anisotropic kriging $\theta_{j}$ is different in each direction. In Table 1, examples of kernel functions in dimension 1 are given [30].

To construct a kriging meta-model, $N$ evaluations of the model are required, i.e. a set of inputs $\left\{\mathbf{x}^{(1)}, \ldots, \mathbf{x}^{(N)}\right\}$ and outputs $\mathbf{y}=\left\{y^{(1)}, \ldots, y^{(N)}\right\}$, called learning set or experimental design (ED). From this set, the regression matrix $\mathbf{G}$ of coefficients $G_{i j}=g_{j}\left(x^{(i)}\right)$ can be constructed as well as the matrix $\mathbf{R}$ of coefficients $R_{i j}=\mathcal{R}\left(\boldsymbol{\theta}, \mathbf{x}^{(i)}, \mathbf{x}^{(j)}\right)$. Then, three parameters must be determined, namely the regression vector $\boldsymbol{\beta}$, the process variance $\sigma^{2}$ and the hyperparameter $\boldsymbol{\theta}$. The first step consists in the determination of $\boldsymbol{\theta}$ by maximizing the likelihood function $L[16,29]$ :

$L=\frac{1}{(2 \pi)^{n / 2)}|\mathbf{C}|^{1 / 2}} \exp \left(-\frac{1}{2}(\mathbf{y}-\mathbf{G} \boldsymbol{\beta})^{\mathrm{T}} \mathbf{C}^{-1}(\mathbf{y}-\mathbf{G} \boldsymbol{\beta})\right)$,

where $\mathbf{C}=\sigma^{2} \mathbf{R}$ and the estimation of $\boldsymbol{\beta}$ is $\boldsymbol{\beta}=\left(\mathbf{G}^{\mathrm{T}} \mathbf{R G}\right)^{-1} \mathbf{G}^{\mathrm{T}} \mathbf{R}^{-1} \mathbf{y}$ and the estimation of the process variance is $\sigma^{2}=\frac{1}{N}(\mathbf{y}-\mathbf{G} \boldsymbol{\beta})^{\mathrm{T}} \mathbf{R}^{-1}(\mathbf{y}-\mathbf{G} \boldsymbol{\beta})$.

The kriging predictor at a new point $\mathbf{x}_{0}$ is then given by $[16,29]$

$\widehat{h}\left(\mathbf{x}_{0}\right)=\mathbf{g}_{0}^{\mathrm{T}} \boldsymbol{\beta}+\mathbf{r}_{0}^{\mathrm{T}} \mathbf{R}^{-1}(\mathbf{y}-\mathbf{G} \boldsymbol{\beta})$,

where $\mathbf{g}_{0}$ is the vector of $g_{j}\left(\mathbf{x}_{0}\right)$ and $\mathbf{r}_{0}$ is the vector of $\mathcal{R}\left(\boldsymbol{\theta}, \mathbf{x}^{(i)}, \mathbf{x}_{0}\right)$.

\subsection{Polynomial Chaos Expansion}

For random variables, stochastic modelling is particularly adapted as it is based on the stochastic properties of the random variable. According to the Polynomial Chaos theory, the second-order random-parameter function $Y(\xi)$, which depends on $\xi$ the vector of $m$ independent random variables $\left(\xi_{i}\right)_{i \in[1, m]}$, can be approximated by a convergent expansion $[19,20,31,32]$ :

$Y(\xi) \approx \sum_{J=0}^{P-1} a_{J} \Psi_{J}(\xi)$,

where $\Psi_{J}$ are the multivariate orthogonal polynomials and $\left(a_{J}\right)_{J \in[0, P-1]}$ are the weighting coefficients to be determined. Each random variable $\left(\xi_{i}\right)$ is described by its PDF $f_{i}$ and the joined PDF of $\xi$ is $f=\prod_{i=1}^{m} f_{i}$. The multivariate polynomials $\Psi_{J}$ are obtained by the tensorization of orthogonal mono-variate polynomials $\left(P_{j}^{(i)}\right)$ of order $j$ and orthogonal to the PDF $f_{i}$. It writes $[19,20,31,32]$

$\Psi_{J}(\xi)=\prod_{i=1}^{m} P_{J_{i}}^{(i)}\left(\xi_{i}\right)$, 
Table 2 Orthogonal polynomial basis from the Askey Scheme [20,31]

\begin{tabular}{lll}
\hline Distribution & Support & Polynomial Chaos basis \\
\hline Uniform & {$[-1,1]$} & Legendre \\
Gaussian & $\mathbb{R}$ & Hermite \\
Beta & {$[0,1]$} & Jacobi \\
Gamma & {$[0,+\infty]$} & Laguerre \\
Chebychev & {$[-1,1]$} & Chebychev \\
\hline
\end{tabular}

where $J=\sum_{i=1}^{m} J_{i}$ is the order of $\Psi_{J}$. The inner product between the two polynomials $P_{i}$ and $P_{j}$ that depends on a random variable $X$ of $\operatorname{PDF} f_{X}$ is given by

$\left\langle P_{i}, P_{j}\right\rangle=\int_{\Omega_{X}} P_{i}(X) P_{j}(X) f_{X}(X) \mathrm{d} X$.

If $c_{o}$ is the chaos order, i.e. the maximum order of polynomials kept in the expansion, then $P=\left(\begin{array}{c}m+c_{o} \\ c_{o}\end{array}\right)$. The sets of polynomials $\left(P_{j}^{(i)}\right)$ are chosen based on the Askey scheme that gives a correspondence between classical PDF and the associated orthogonal polynomial family [20,31]. This is illustrated in Table 2 for a few families. The number of terms in the PCE can increase drastically when high chaos order is considered or when the number of random parameters increases. To limit the number of terms in the expansion and avoid convergence issues, some truncation strategies are possible [33], here the hyperbolic truncation norm is adopted to select the polynomials to keep [34]. It is based on the idea that models and physics are often driven by low-order interaction effects. The polynomials that are kept in Eq. (6) verify

$\|J\|_{\gamma}=\left(\sum_{j=1}^{m} J_{j}^{\gamma}\right)^{1 / \gamma} \leq c_{o}$

where $\gamma \in(0,1]$ is the chosen hyperbolic norm coefficient. The coefficients $\left(a_{J}\right)_{J \in[0, P-1]}$ can be computed based on intrusive and non-intrusive approaches. The non-intrusive approach based on the regression method has proved to be efficient $[32,34]$ and is used here. The $\left(a_{J}\right)_{J \in[0, P-1]}$ are computed by minimizing in the least-square sense the distance between the random function $Y(\xi)$ and and its PCE evaluations at $M$ points.

\subsection{Hybrid approach coupling kriging and PCE}

In this section, the hybrid method that couples kriging and PCE to deal with both random and parametric parameters is presented [28]. As previously explained, the objective is to create a surrogate function $\widehat{f}(\mathbf{x}, \boldsymbol{\xi})$ of $f(\mathbf{x}, \boldsymbol{\xi})$ where $\mathbf{x}$ is a vector of parametric variables of dimension $n$ and $\xi$ the vector of random parameters of dimension $m$. First, the random part is decomposed using the PCE approximation:

$f(\mathbf{x}, \boldsymbol{\xi}) \approx \widehat{f}(\mathbf{x}, \boldsymbol{\xi})=\sum_{J=0}^{P-1} a_{J}(\mathbf{x}) \Psi_{J}(\boldsymbol{\xi})$,

where the chaos coefficients $\left(a_{J}\right)_{J \in[0, P-1]}$ now depend on the parametric variables $\mathbf{x}$. Each PCE coefficient is then approximated based on a kriging meta-model:

$a_{J}=\mathbf{g}^{(J)}(\mathbf{x})^{\mathrm{T}} \boldsymbol{\beta}^{(J)}+\mathcal{Z}^{(J)}(\mathbf{x})$.

Finally, it comes

$f(\mathbf{x}, \boldsymbol{\xi}) \approx \widehat{f}(\mathbf{x}, \boldsymbol{\xi})=\sum_{J=0}^{P-1}\left(\mathbf{g}^{(J)}(\mathbf{x})^{\mathrm{T}} \boldsymbol{\beta}^{(J)}+\mathcal{Z}^{(J)}(\mathbf{x})\right) \Psi_{J}(\boldsymbol{\xi})$. 
Generate a LHS of $M$ values of $\boldsymbol{\xi}$

$$
\Xi=\left(\boldsymbol{\xi}^{(1)}, \ldots, \boldsymbol{\xi}^{(M)}\right)
$$

Evaluate the expensive model $f$ for the $N \times M$ configurations of $\boldsymbol{\xi}$ and $\mathbf{x}$

Generate a LHS of $N$ values of $\mathbf{x}$

$$
\mathbf{X}=\left(\mathbf{x}^{(1)}, \ldots, \mathbf{x}^{(N)}\right)
$$

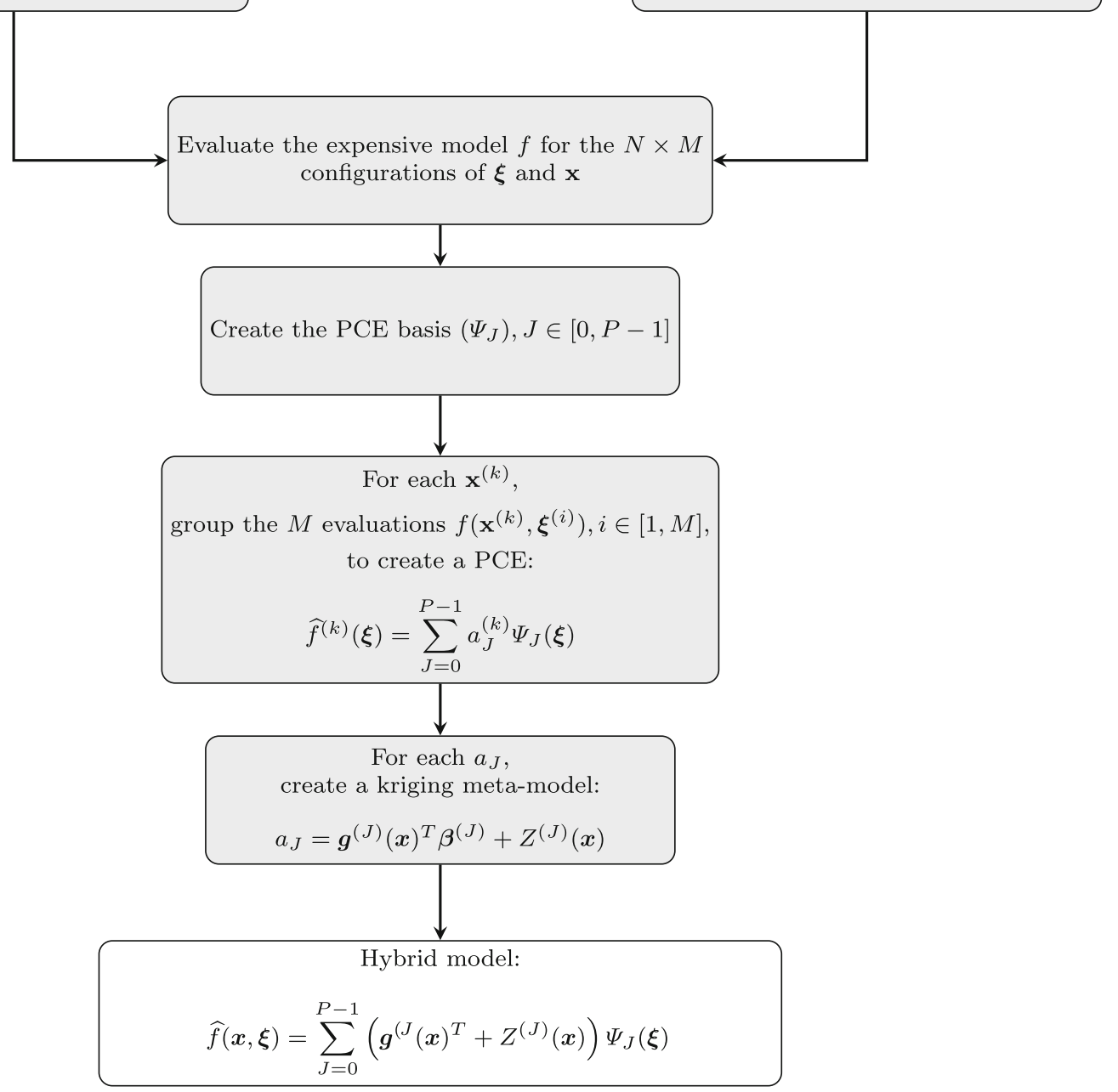

Fig. 1 General workflow of the method

The construction of the experimental design is based on a set of a limited number of evaluations of the function $f$. Two input sets are generated: a first one of $N$ values $\mathbf{x}^{(i)}$ and a second of $M$ values $\boldsymbol{\xi}^{(j)}$, the final input set is obtained by tensorization of these two sets (i.e. $N \times M$ values). The model is evaluated for all these points. For each $\mathbf{x}^{(i)}, M$ evaluations of $f$ are available from the different $\xi^{(j)}$ and a PCE is constructed. As $N$ PCE are constructed, $N$ values of each $\left(a_{J}\right)_{J \in[0, P-1]}$ are available and used to construct a kriging meta-model. The general workflow is illustrated in Fig. 1.

\subsection{Exploitation of the coefficients}

The PCE decomposition gives a direct access to many statistical information without any additional simulations, that are directly dependent on the parametric variables here, which is the main interest of the approach. Indeed, if a unique kriging meta-model were build, then it would have been necessary to perform an MCS simulation. Thus, 

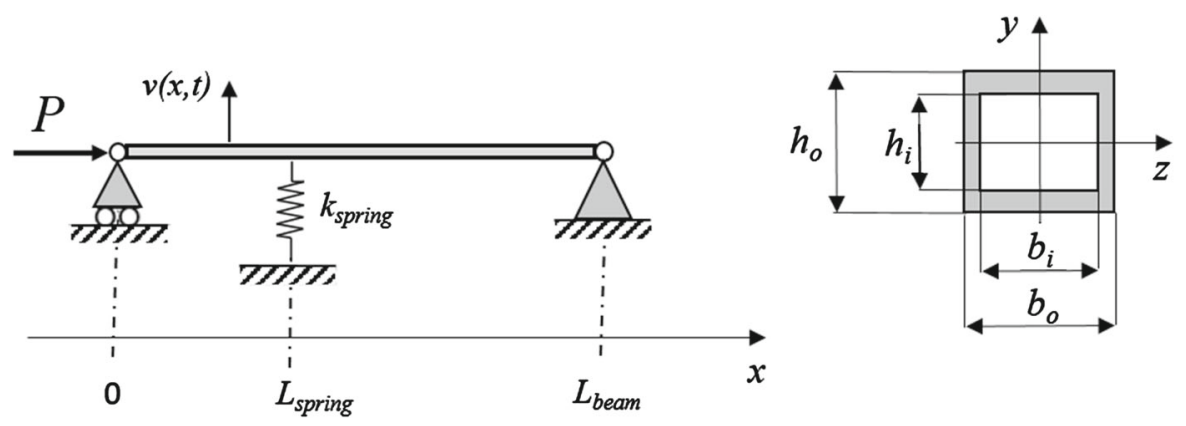

Fig. 2 Mechanical system under study

the average of $f$ at the points $\mathbf{x}$ is given by

$\mathbb{E}[f(\mathbf{x})]=a_{0}=\mathbf{g}^{(0)}(\mathbf{x})^{\mathrm{T}} \boldsymbol{\beta}^{(0)}+\mathcal{Z}^{(0)}(\mathbf{x})$

and the variance is given by

$\sigma_{f(\mathbf{x})}^{2}=\sum_{J=1}^{P-1} a_{J}(\mathbf{x})^{2}\left\|\Psi_{J}\right\|^{2}=\sum_{J=1}^{P-1}\left(\mathbf{g}^{(J)}(\mathbf{x})^{\mathrm{T}} \boldsymbol{\beta}^{(J)}+\mathcal{Z}^{(J)}(\mathbf{x})\right)^{2}\left\|\Psi_{J}\right\|^{2}$.

The Sobol indices can also be directly estimated from the PCE decomposition due to its uniqueness. If $V(f(\mathbf{x}))$ is the variance of the output at the point $\mathbf{x}$, then the first-order Sobol index $S_{i}$ related to the variable $\xi_{i}$ is [35]

$S_{i}(\mathbf{x})=\frac{V_{i}(\mathbf{x})}{V(f(\mathbf{x}))}$,

where $V(\mathbf{x})=V\left(\mathbb{E}\left(f(\mathbf{x}) \mid X_{i}\right)\right.$. The latter is directly deduced from the PCE coefficients [32]:

$V_{i}(\mathbf{x})=\sum_{J \in \boldsymbol{\alpha}_{i}} a_{J}(\mathbf{x})^{2}\left\|\Psi_{J}\right\|^{2}$,

where $\boldsymbol{\alpha}_{i}$ is the set of the multivariate indices for which only polynomials related to the variable $\xi_{i}$ are present.

\section{Mechanical system under study}

\subsection{Equations of motion}

The mechanical system under study is shown in Fig. 2. It is composed of a beam of length $L_{\text {beam }}$ and of rectangular cross section with inner sides $b_{i}$ and $h_{i}$ and external sides $b_{o}$ and $h_{o}$. The system is simply supported at both ends by rigid supports and at position $x=L_{\text {spring }}$ by a spring of stiffness $k_{\text {spring. The beam is loaded by a uniform axial }}$ force $P$ which acts on the beam neutral axis. The beam deflection is described by a deflection function $v(x, t)$ (i.e. amount of vertical displacement at position $x$ on the beam from the left end).

In the following, the classical Rayleigh-Ritz method is proposed to formulate the mathematical problem and obtain the equations of motion of the studied system. As a reminder, the Rayleigh-Ritz approach that corresponds to an approximate energy-based method has been widely used to solve problems of solid mechanics and more particularly to investigate the dynamics (such as the natural frequencies and mode shapes) as well as the buckling behaviour of vibrating beam structures [36]. Using the Rayleigh-Ritz method, the displacement function $v(x, t)$ of the beam is approximated as a linear combination of $n$ trial functions $\phi_{i}(x)$ such as

$v(x, t) \approx \sum_{i=1}^{n} \phi_{i}(x) \lambda_{i}(t)$ 
where $\lambda_{i}(t)$ are arbitrary coefficients. Each trial function $\phi_{i}$ should satisfy at least the essential boundary conditions (i.e. geometrical boundary conditions). In the present case, these two boundary conditions are given by zero displacement at the right-hand and left-hand supports: $v(0)=0$ and $v\left(L_{\text {beam }}\right)=0$. It is to be noted that the use of polynomials or trigonometric series is classically used in the modelling of many physical problems such as buckling and vibration. Finally, the original variational problem of extremizing the functional integral $F[v(x, t)]$ is replaced by the problem of finding the coefficients $\lambda_{i}$ that extremizes $F\left(\lambda_{i}, \ldots, \lambda_{n}\right)$. So minimizing the energy functional $F$ can be done by taking partial derivatives with respect to $\lambda_{i}$ such as $\frac{\partial F}{\partial \lambda_{i}}=0$. The convergence of the Rayleigh-Ritz process implies that the approximation given in Eq. (17) will tend towards the exact deflection function $v(x, t)$ that extremizes the integral $F[v(x, t)]$ when $n$ tends to infinity.

For the beam system under study, the maximum kinetic energy $T$ is given by

$T=\frac{1}{2} \int_{0}^{L_{\text {beam }}} \rho S\left(\frac{\partial v(x, t)}{\partial t}\right)^{2} \mathrm{~d} x$,

where $\rho$ and $S$ define the beam density and the cross-sectional area (i.e. $S=b_{e} h_{e}-b_{i} h_{i}$ ), respectively.

The maximum strain energy $V$ is composed of the contributions of the beam and the punctual spring $k_{\text {spring }}$ such as

$V=\frac{1}{2} \int_{0}^{L_{\text {beam }}} E I\left(\frac{\partial^{2} v(x, t)}{\partial^{2} x}\right)^{2} d x+\frac{1}{2} k_{\text {spring }}\left(v\left(L_{\text {spring }}, t\right)\right)^{2}$,

where $E$ and $I$ define the Young's modulus of the beam and the second moment of area of the beam cross section (i.e. $\left.I=\frac{b_{e} h_{e}^{3}-b_{i} h_{i}^{3}}{12}\right)$, respectively.

Then the amount of work $W_{P}$ performed by the external concentrated load $P$ from the initial position to the bent situation is equal to

$W_{P}=-P v_{P}$,

where $v_{P}$ corresponds to the horizontal displacement generated by the load $P$. By using a Taylor expansion and neglecting the higher order terms, the horizontal displacement $v_{P}$ can be approximated by [1]

$v_{P}=\frac{1}{2} \int_{0}^{L_{\text {beam }}}\left(\frac{\partial v(x, t)}{\partial x}\right)^{2} \mathrm{~d} x$.

By equating the total potential energy $\Pi=-T+V+W_{P}$ and substituting $v(x, t)$ by the approximate admissible displacement provided in Eq. (17), the equation of motion with respect to the generalized coordinates $\lambda_{i}$ is given by

$\mathbf{M} \ddot{\Lambda}(t)+\left(\mathbf{K}-\mathbf{K}_{P}\right) \Lambda(t)=\mathbf{0}$

with

$$
\begin{aligned}
& \Lambda(t)=\left(\lambda_{1}, \ldots, \lambda_{n}\right)^{\mathrm{T}}, \\
& \mathbf{M}_{i j}=\rho S \int_{0}^{L_{\text {beam }}} \phi_{i}(x) \phi_{j}(x) \mathrm{d} x, \\
& \mathbf{K}_{i j}=E I \int_{0}^{L_{\text {beam }}} \frac{\partial^{2} \phi_{i}(x)}{\partial^{2} x} \frac{\partial^{2} \phi_{j}(x)}{\partial^{2} x} \mathrm{~d} x+k_{\text {spring }} \phi_{i}\left(L_{\text {spring }}\right) \phi_{j}\left(L_{\text {spring }}\right), \\
& \mathbf{K}_{P, i j}=P \int_{0}^{L_{\text {beam }}} \frac{\partial \phi_{i}(x)}{\partial x} \frac{\partial \phi_{j}(x)}{\partial x} \mathrm{~d} x
\end{aligned}
$$

for $i, j=1, \ldots, n$.

Considering the essential boundary conditions to be verified, a linear combination of sinusoidal functions are applied for approximating the displacement function $v(x, t)$ :

$\phi_{i}(x)=\sin \left(\frac{i \pi x}{L_{\text {beam }}}\right), \quad i=1, \ldots, n$. 
Substituting Eq. (27) in Eqs. (24), (25) and (26), the mass matrix $\mathbf{M}$ and the stiffness matrices $\mathbf{K}$ and $\mathbf{K}_{P}$ are given by

$$
\begin{aligned}
& \mathbf{M}=\rho S \frac{L_{\text {beam }}}{2} \mathbf{I}, \\
& \mathbf{K}_{i j}=\left\{\begin{array}{l}
\frac{E I(i \pi)^{4}}{2 L_{\text {beam }}^{3}}+k_{\text {spring }}\left(\sin \left(\frac{i \pi L_{\text {spring }}}{L_{\text {beam }}}\right)\right)^{2} \text { if } i=j, \\
k_{\text {spring }} \sin \left(\frac{i \pi L_{\text {spring }}}{L_{\text {beam }}}\right) \sin \left(\frac{j \pi L_{\text {spring }}}{L_{\text {beam }}}\right) \text { if } i \neq j,
\end{array}\right. \\
& \mathbf{K}_{P, i j}=\left\{\begin{array}{cc}
\frac{P(i \pi)^{2}}{2 L_{\text {beam }}} & \text { if } i=j, \\
0 & \text { if } i \neq j,
\end{array}\right.
\end{aligned}
$$

for $i, j=1, \ldots, n$.

Assuming a solution of the form $\Lambda(t)=\mathbf{u} e^{\mathrm{i} \omega t}$ (where $\omega$ corresponds to the angular frequency of the system and $\mathbf{u}$ the associated eigenvector in the generalized coordinates $\lambda_{i}$ ), system (22) becomes

$$
\left(-\omega^{2} \mathbf{M}+\mathbf{K}-\mathbf{K}_{P}\right) \mathbf{u}=\mathbf{0} .
$$

Eigenvalues are determined by solving the associated characteristic equation (i.e. $\operatorname{det}\left(-\omega^{2} \mathbf{M}+\mathbf{K}-\mathbf{K}_{P}\right)=0$ ). It is recalled that each angular frequency $\omega_{i}$ resulting from the discretization of the displacement variational principle by the Rayleigh-Ritz method is an upper bound to the corresponding exact angular frequency $\omega_{i}^{e}$ (i.e. $\omega_{i}^{e} \leq \omega_{i}$ for $i=1, \ldots, n)$.

It is often very difficult to determine the exact critical buckling load in structures using the classical Euler's theory. Therefore, the estimation of buckling loads is often approximated using energy conservation and referred to as an energy method in structural analysis. The principle of stationary potential energy can be used to find the critical loads by considering the total potential energy given by $\Pi=V+W_{P}$ (i.e. at the critical load, the neutral equilibrium is possible, therefore, $\frac{\Pi}{P}=0$ ). It corresponds to the simplification of the eigenproblem previously discussed (see Eq. (31)) to its restriction to the sudden change in shape (deformation) of the beam under load. For a linear buckling analysis, the eigenvalue problem to be solved to get the $j$ th buckling load multiplier $\alpha_{j}$ and the associated buckling mode shapes $\Psi_{j}$ is defined by $\left(\mathbf{K}-\alpha_{j} \mathbf{K}_{P}\right) \Psi_{j}=\mathbf{0}$. The $j$ th buckling load is then provided by $P_{j}^{b}=\alpha_{j} P$. It is to be noted that buckling load multiplier is an indicator of the factor of safety against buckling: if $\alpha_{j}>1$ the initial applied load is less that the estimated critical buckling load $P_{j}^{b}$ and buckling are not predicted. The natural frequencies of the mechanical system with the axial applied load $P$ are provided by Eq. (31). If $\alpha_{j}<1$ the initial applied load exceeds the estimated critical buckling load $P_{j}^{b}$ and buckling will occur. For $\alpha_{j}=1$ the applied load is exactly the critical buckling load $P_{j}^{b}$ and buckling is expected. The critical loads obtained will be an approximate value of the exact critical loads due to the fact that the exact deflected shape is not available. The approximated deflected shape assumed is provided by Eq. (17). Moreover, it is worth mentioning that in the present case, the presence of a spring with variable position and intensity makes impossible an analytical expression of the buckling loads and therefore requires numerical processing. Therefore, taking into account uncertainties (i.e. random geometric imperfections and fluctuations in material properties) for the prediction of buckling loads requires an efficient numerical methodology.

\subsection{Preliminary deterministic study}

First of all, evolutions of the first three natural frequencies of the beam system subjected to the load $P$ are displayed in Fig. 3a for different values of both the stiffness $k_{\text {spring }}$ and position of the spring $L_{\text {spring. The values of the physical }}$ parameters used for the beam system are indicated in Table 3 . It appears very clearly that each frequency has a potentially very different evolution from the others depending on the two parameters of the spring $\left(k_{\text {spring }}, L_{\text {spring }}\right)$. 
Table 3 Geometrical and physical parameters of the beam

\begin{tabular}{lc}
\hline Parameter & Value \\
\hline Inner height $h_{i}(\mathrm{~m})$ & 0.06 \\
Inner base $b_{i}(\mathrm{~m})$ & 0.06 \\
Outer height $h_{o}(\mathrm{~m})$ & 0.1 \\
Outer base $b_{o}(\mathrm{~m})$ & 0.1 \\
Length $L_{\text {beam }(\mathrm{m})}$ & 1 \\
Young's modulus $E(\mathrm{~Pa})$ & $2 \times 10^{11}$ \\
Density $\rho\left(\mathrm{kg} / \mathrm{m}^{3}\right)$ & 7800
\end{tabular}

(a)

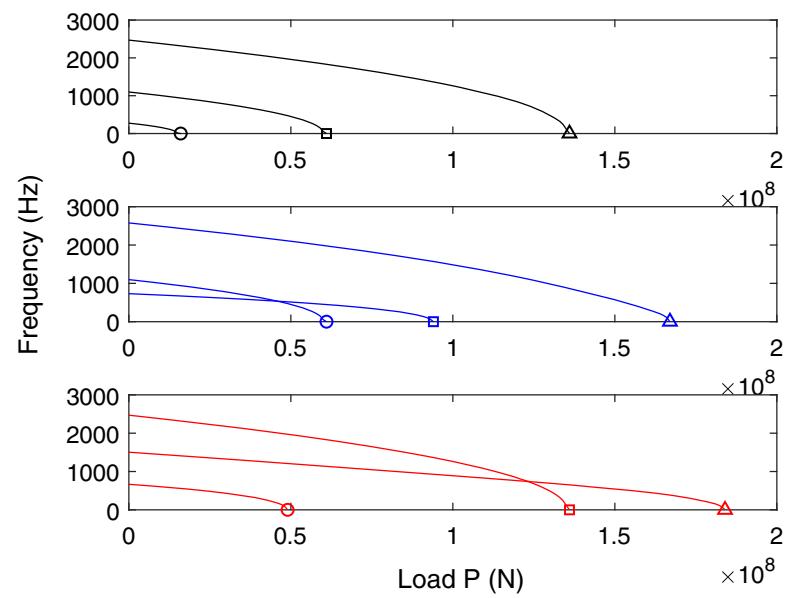

(b)

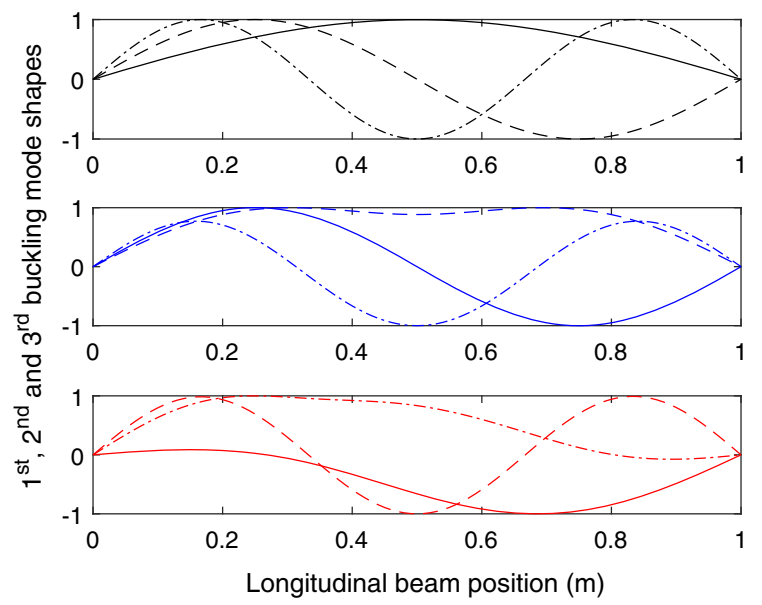

Fig. 3 Effect of $\left(k_{\text {spring }}, L_{\text {spring }}\right)$ on (a) frequencies evolution and buckling loads (black: no spring ; blue: $k_{\text {spring }}=10^{9} \mathrm{~N} / \mathrm{m}$ and $L_{\text {spring }}=0.5 \mathrm{~m}$; red: $k_{\text {spring }}=5.10^{8} \mathrm{~N} / \mathrm{m}$ and $L_{\text {spring }}=0.33 \mathrm{~m} ; \circ 1$ st buckling load; $\square$ 2nd buckling load; $\Delta 3^{\text {rd }}$ buckling load) and on (b) the buckling mode shapes (-1st, - - - 2nd and -...- 3rd)

One can more particularly observe in certain cases a crossing of two frequencies when the value of the applied load $P$ increases (see crossing between the first and second frequencies (second case, in blue) and crossing between the second and third frequencies (third case, in red)). This well-known effect in the field of vibrations is due to the position of the spring $L_{\text {spring }}$ in regard to the nodes and anti-nodes of mode shapes: if the spring is close to a node its influence will be less important than if it is located close to an anti-node (location where the amplitude is maximum). Of course, for a given position $L_{\text {spring }}$ the increase of the stiffness $k_{\text {spring }}$ leads to an increase of the frequencies (except if the spring is located on a node of the associated mode shape).

It is well known that gradually increasing the load $P$ decreases the natural frequencies of the beam system. When the load reaches a critical level, the beam system may suddenly change its shape. This phenomenon called buckling corresponds to the fact that a natural frequency of the system has reached a zero value. For example, the first three buckling loads $P_{i}^{b}$ (for $i=1, \ldots, 3$ ) are identified by a circle $\circ$, a square $\square$ and a triangle $\Delta$ in Fig. $3 \mathrm{a}$. The associated buckling mode shapes are also given in Fig. 3b. Once again these results clearly demonstrate the impact of the two spring parameters $\left(k_{\text {spring }}, L_{\text {spring }}\right)$ on the value of the buckling loads and also on the fundamental and second buckling modes. In conclusion, the eigenfrequencies, the associated eigenmodes as well as the buckling loads of the mechanical system are dependent on the combination $\left(P, k_{\text {spring }}, L_{\text {spring }}\right)$.

In order to have a more global overview of the influence of the two parameters $\left(k_{\text {spring }}, L_{\text {spring }}\right)$ on the first two buckling loads $P_{1}^{b}$ and $P_{2}^{b}$, simulations are conducted for $100 \times 100$ combinations of these two deterministic inputs 


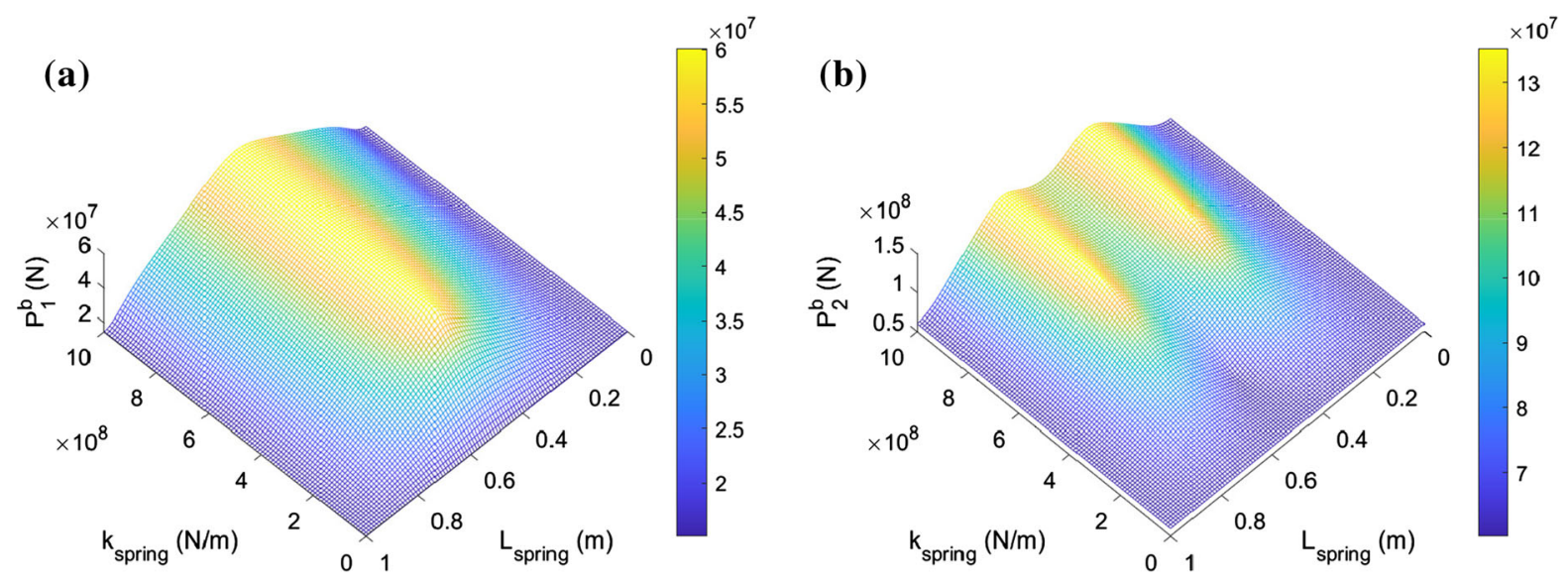

Fig. 4 Evolution of the first two buckling loads (a) $P_{1}^{b}$ and (b) $P_{2}^{b}$ versus $\left(k_{\text {spring }}, L_{\text {spring }}\right)$

by sampling the two-dimensional space parameter $\left(k_{\text {spring }}, L_{\text {spring }}\right)$ for $k_{\text {spring }} \in\left[0 ; 10^{9}\right] \mathrm{N} / \mathrm{m}$ and $L_{\text {spring }} \in[0 ; 1] \mathrm{m}$. For each deterministic couple $\left(k_{\text {spring }}, L_{\text {spring }}\right)$, the values of the first two buckling loads $P_{1}^{b}$ and $P_{2}^{b}$ are estimated and the results are illustrated in Fig. 4. It is to be noted that the proposed results have been performed by approximating the displacement function $v(x, t)$ of the beam by a combination of 50 trial functions $\phi_{i}(x)$ as previously explained in Sect. 3.1 (see Eq. (17)). A convergence study has also been conducted in order to verify the convergence of the results on the estimation of the first two buckling loads as a function of the number of functions selected in the Rayleigh-Ritz method. Figure 5 give the two values $\Delta_{\max }^{i, n}$ and $\Delta_{\text {mean }}^{i, n}$ defined by

$$
\begin{aligned}
& \Delta_{\text {max }}^{i, n}=\max _{\substack{k_{\text {spring }} \in\left[0 ; 10^{9}\right] N \\
L_{\text {spring }} \in[0 ; 1] m}}\left(\frac{\left|P_{i, \text { ref }}^{b}-P_{i, n}^{b}\right|}{P_{i, \text { ref }}^{b}}\right), \\
& \Delta_{\text {mean }}^{i, n}=\operatorname{mean}_{\substack{k_{\text {spring }} \in\left[0 ; 10^{9}\right] N \\
L_{\text {spring }} \in[0 ; 1] m}}\left(\frac{\left|P_{i, \text { ref }}^{b}-P_{i, n}^{b}\right|}{P_{i, \text { ref }}^{b}}\right),
\end{aligned}
$$

where $P_{i \text {,ref }}^{b}$ defines the $i$ th reference buckling load (chosen for $n=50$ ) and $P_{i, n}^{b}$ corresponds to the $i$ th approximate buckling load based on $n$ trial functions (with $n \leq 30$ ). These two indicators $\Delta_{\text {max }}^{i, n}$ and $\Delta_{\text {mean }}^{i, n}$ are estimated by considering the $100 \times 100$ calculations in regard to the two deterministic inputs $\left(k_{\text {spring }}, L_{\text {spring }}\right)$. As shown in Fig. 5, using 10 functions $\phi_{i}(x)$ allows to have a mean error $\Delta_{\text {mean }}^{i, n}$ of the global estimate lower than $0.02 \%$ and a maximum error $\Delta_{\text {max }}^{i, n}$ lower than $0.1 \%$ for both the first and second buckling loads $P_{1}^{b}$ and $P_{2}^{b}$. Coming back briefly to Fig. 4 , the buckling loads evolutions are symmetric about $L_{\text {spring }}=0.5 \mathrm{~m}$. The first buckling load is maximal when the spring is located at the centre of the beam, whereas for the second buckling load the maximum is reached when the spring is at the third or two-third of the length of the beam. The buckling loads tend to increase when the spring stiffness increases as well until a threshold is reached. The latter is reached at about $2.5 \times 10^{8} \mathrm{~N} / \mathrm{m}$ for the first buckling load, and at about $6.4 \times 10^{8} \mathrm{~N} / \mathrm{m}$ for the second buckling load.

Finally, it is important to remember that many physical parameters of the beam system can have a significant influence on the buckling loads. Some parameters may vary throughout the life of the mechanical system or some uncertainties have to be taken into account during the manufacturing process. All of these facts can affect the estimation of buckling loads and their impacts must therefore be quantified. However, conducting such a study requires a considerable number of simulations, resulting in excessive calculation times. As a result, it is necessary to deploy new strategies in order to considerably reduce the number of calculations and to respond to this current problem. The main objective of the rest of study is to promote the use of PCE-kriging surrogate model and to 
(a)
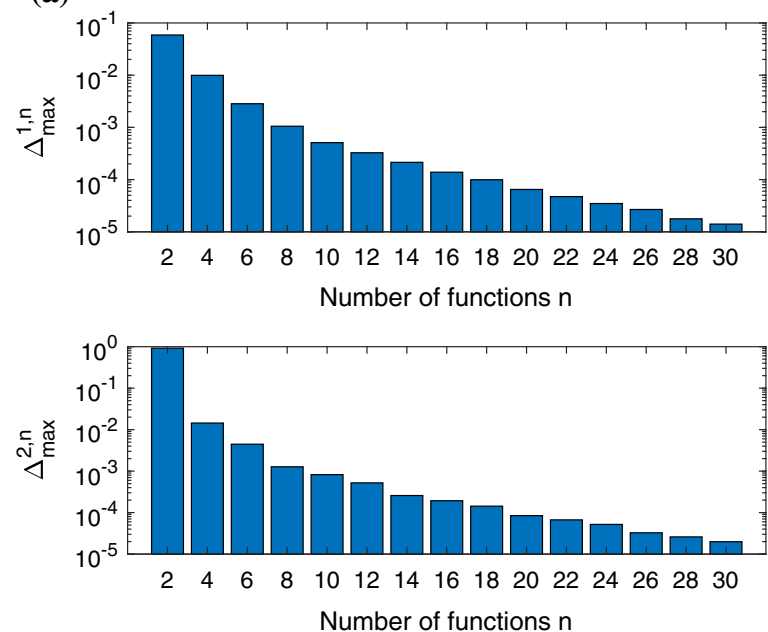

(b)
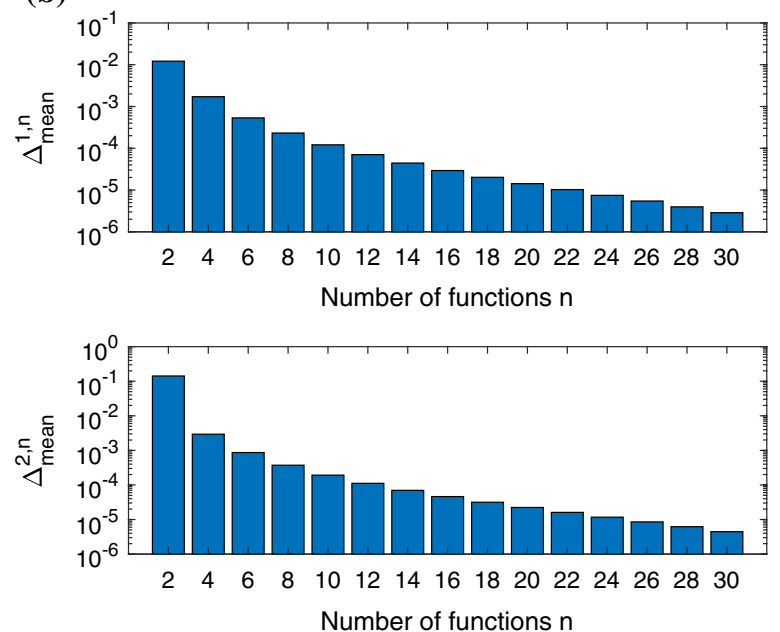

Fig. 5 Evolution of (a) $\Delta_{\max }^{i, n}$ and (b) $\Delta_{\text {mean }}^{i, n}$ for the first two buckling loads

Table 4 Random parameters characteristic

\begin{tabular}{lllll}
\hline Name & Notation & Distribution & Mean & Standard deviation \\
\hline Young modulus & $E$ & Normal & $2 \times 10^{11} \mathrm{~Pa}$ & $3.5 \times 10^{9} \mathrm{~Pa}$ \\
Beam length & $L_{\text {beam }}$ & Normal & $1 \mathrm{~m}$ & $0.0167 \mathrm{~m}$ \\
Outer height & $h_{o}$ & Normal & $0.1 \mathrm{~m}$ & $0.017 \mathrm{~m}$ \\
Outer base & $b_{o}$ & Normal & $0.1 \mathrm{~m}$ & $0.017 \mathrm{~m}$ \\
Inner height & $h_{i}$ & Normal & $0.06 \mathrm{~m}$ & $8 \times 10^{-4} \mathrm{~m}$ \\
Inner base & $b_{i}$ & Normal & $0.06 \mathrm{~m}$ & $8 \times 10^{-4} \mathrm{~m}$ \\
\hline
\end{tabular}

illustrate its efficiency to accurately predict the first two buckling loads $P_{1}^{b}$ and $P_{2}^{b}$ of the beam system with multiple uncertainties.

\section{Application and numerical examples}

In this section, the PCE-kriging approach is employed to predict the two first buckling loads $P_{1}^{b}$ and $P_{2}^{b}$ when the uncertainties present in the model are considered. In the present study, all the model parameters are considered as uncertain, and are described by a normal distribution. It represents a total of six uncertain parameters: the Young modulus $E$, the beam length $L_{\text {beam }}$, the inner base $b_{i}$, the inner height $h_{i}$, the outer base $b_{o}$ and the outer height $h_{o}$. Their characteristics (mean and standard deviation) are given in Table 4 . The choice was done to ensure that $95 \%$ of the samples represent a 5\% variation around the mean value for each parameter. It is worth noticing that the density is not considered as random, as it has no influence on $P_{1}^{b}$ and $P_{2}^{b}$. To be noted that the effect of boundary conditions is not investigated in the proposed study. The system is thus subjected to two types of varying parameters: a parametric variation of the spring location $L_{\text {spring }}$ and stiffness $k_{\text {spring }}$, and the random aspect of all the parameters that characterize the beam. To treat with these two natures of parameter, the PCE-kriging approach is employed. 


\subsection{Construction of the PCE-kriging meta-models}

As a first step, the experimental design is constructed. As the system is symmetric about $L_{\text {spring }}$, the location of $L_{\text {spring }}$ is taken between 0 and $0.5 L_{\text {beam. }}$. A set of 50 points generated by an LHS of dimension 6 is taken for the PCE construction, and a set of 51 points constructed by a LHS of dimension 2 that satisfies a maximin criterion is created for the kriging. Finally, the buckling loads $P_{1}^{b}$ and $P_{2}^{b}$ are computed for the $50 \times 51=2550$ configurations of the system.

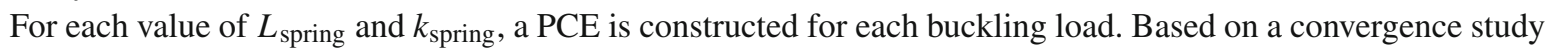
realized for different spring properties and not presented here for the sake of concision, the chaos order is taken equal to 5 and a 0.5 -hyperbolic norm is chosen, so the PCE basis is composed of 40 terms. The PCE coefficients are computed based on the regression approach for each buckling load and for each value of $L_{\text {spring }}$ and $k_{\text {spring. At this }}$ stage, a first validation step has been undertaken. On a $11 \times 11$ grid of the spring properties (stiffness and location), at each grid point a PCE for each buckling load was created. In addition, for each point of the grid, 1000 random samples were also generated. The PCE predictions were compared to the reference values. In each case, the PCEs have shown good prediction quality, not shown here for the sake of concision.

The second step consists in the construction of the kriging meta-models for each PCE coefficient, i.e. for each of the 40 coefficients of the PCE basis for each load. For the first buckling load $P_{1}^{b}$, a spline correlation with a zero-order polynomial regression is taken, whereas a matérn $3 / 2$ correlation with a zero-order regression kriging is taken for the second buckling load $P_{2}^{b}$.

The final PCE-kriging meta-models are validated by comparing the relative error on the mean and the variance of each buckling load on a $26 \times 51$ grid between the meta-model and reference values. The reference mean and variance are determined from a LHS of 1000 points, which has reached convergence. The evolution of the relative error for the mean and the variance of the first buckling load $P_{1}^{b}$ (resp. $P_{2}^{b}$ ) are given in Fig. 6 (resp. Fig. 7) on the right column. The red points correspond to the 51 points of the kriging-experimental design, i.e. points where PCE are constructed. As a visual indicator of the quality of the meta-models, the average value and variance of each buckling loads of the PCE-kriging meta-models and of the reference are also displayed on the left column. The reference results obtained from Monte Carlo Simulations are given in purple and the PCE-kriging predictions are given in green.

Considering the first buckling load, the relative error is always inferior to $5 \%$ for the average prediction, as illustrated in Fig. 6b, d, demonstrating the high quality of the prediction. Looking at Fig. 6a one can see the very good agreement between the predictions and the reference results. In average, the buckling load $P_{1}^{b}$ increases when

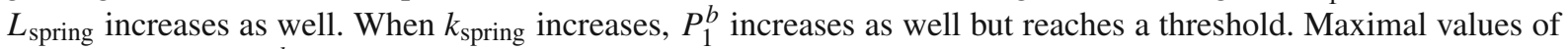
the buckling load $P_{1}^{b}$ are then reached when the spring is fixed at the centre of the beam and when $k_{\text {spring }}$ is larger than $2.5 \times 10^{8} \mathrm{~N} / \mathrm{m}$. For the variance, except a peak of $50 \%$, the relative error is always inferior to $10 \%$ which shows here again the high quality of the PCE-kriging meta-model. The error peak is reached when $L_{\text {spring }} / L_{\text {beam }}=0.5$

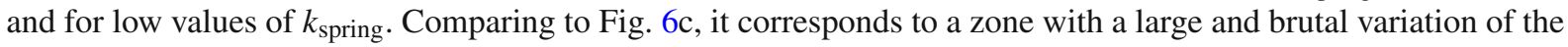
variance. Higher quality of the meta-model could be reached by adding more points in this area. Despite this peak, one can see in Fig. $6 c$ that the variance prediction remains substantially satisfactory. The variance tends to increase when both the spring location and stiffness increase. When the spring is near the base or when the stiffness is low, the variance is low and the buckling load $P_{1}^{b}$ is not significantly influenced by the uncertainties. However, when the spring is getting closer to the centre of the beam or when the spring stiffness increases, the variance increases and the bucking load is significantly impacted by the uncertainty present in the model.

Considering the second buckling load and Fig. 7, the relative error on the average is always inferior to 3\%, as illustrated in Fig. 7b, d, which demonstrates again the accurate prediction of the PCE-kriging meta-model. Looking at the comparison between the predictions of the average buckling load $P_{2}^{b}$ and the reference in Fig. 7a, one can see the good agreement between the two surfaces. The maximum buckling load is reached when the spring is located at the one-third of the beam length (i.e. $L_{\text {spring }} / L_{\text {beam }}=0.33$ or $L_{\text {spring }} / L_{\text {beam }}=0.66$ due to the symmetry). As for the first buckling load, the second one tends to remain constant when the spring is near the base or when the stiffness is low. Similarly, a threshold of the maximum buckling load is reached when $k_{\text {spring }}$ increases. The latter 

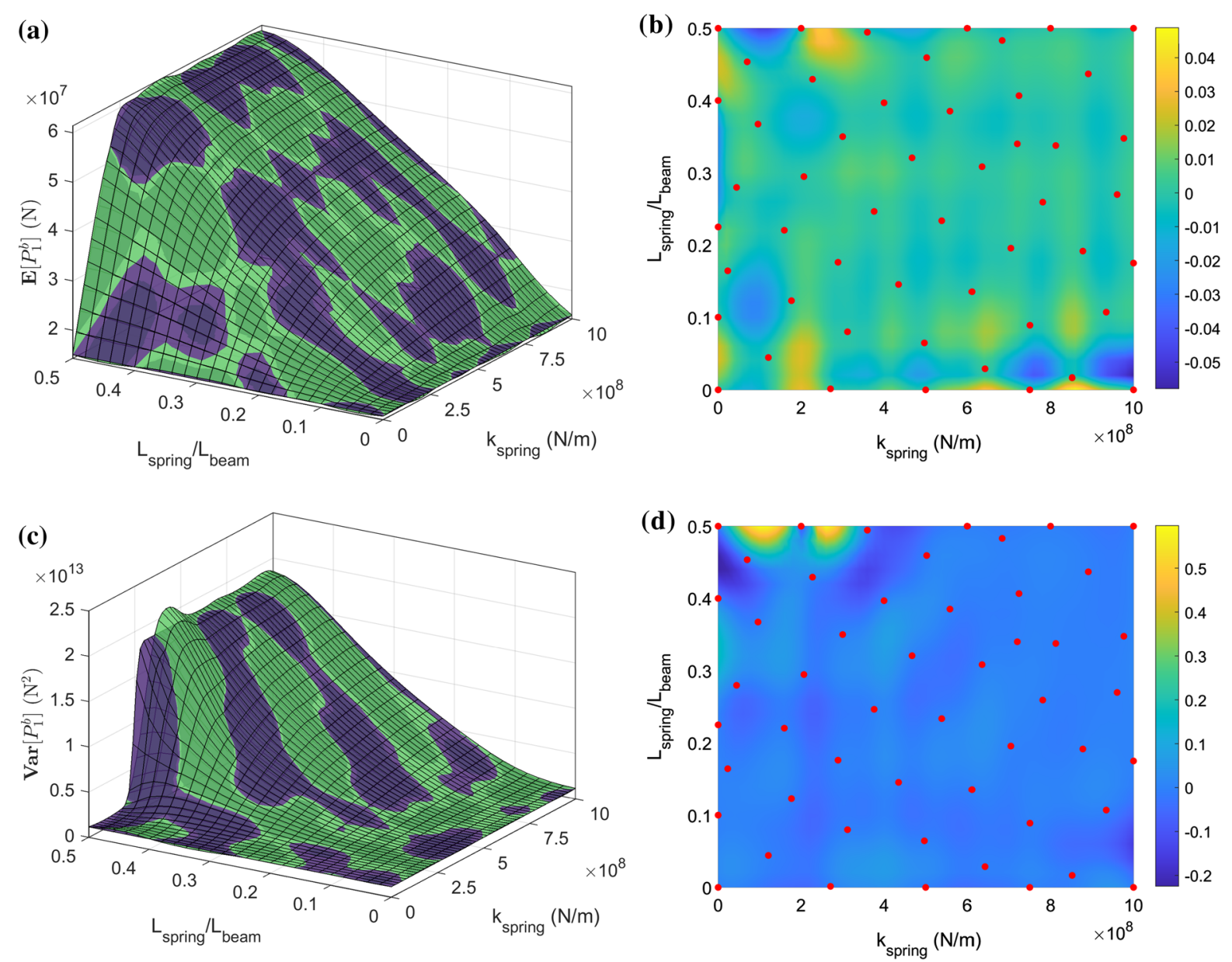

Fig. 6 Buckling load $P_{1}^{b}$-Evolution of the mean (a) and variance (c) for the reference (purple) and PCE-kriging prediction (green) Relative error of the mean (b) and variance $(\mathbf{d})$. Color figure online

is reached later compared to the first buckling load $\left(2.5 \times 10^{8} \mathrm{~N} / \mathrm{m}\right.$ versus $\left.6.4 \times 10^{8} \mathrm{~N} / \mathrm{m}\right)$. Similar analysis can be done for the variance, the relative error is globally low (inferior to 10\%) except at two peaks that correspond to brutal variation of the variance. The variance keeps low value when the spring is near the base or has a low stiffness, and reaches high values when the buckling load is maximal.

It is worth noticing here that the standard deviation corresponds to the square root of the variance and that in the case of a Gaussian distribution, $95 \%$ of the data are in the interval $\left[\overline{P_{i}^{b}}-2 \sigma_{i}, \overline{P_{i}^{b}}+2 \sigma_{i}\right]$, where $\overline{P_{i}^{b}}$ is the average of $P_{i}^{b}$ with $i=1,2$ and $\sigma_{i}$ defined the associated standard deviation. Thus here, it can represent a variation of the buckling load up to about $8.95 \times 10^{6} \mathrm{~N}$ and $1.90 \times 10^{7} \mathrm{~N}$ for $P_{1}^{b}$ and $P_{2}^{b}$ around their average value, respectively, which corresponds to the the maximum of $\left(2 \sigma_{i}\right)$ on the two-dimensional deterministic space inputs (i.e. the couple values $\left.\left(L_{\text {spring }} / L_{\text {beam }}, k_{\text {spring }}\right)\right)$.

It is worth saying a few words here about the computational time related to the approach. First, considering the number of evaluation of the model, with the PCE-kriging meta-model, 2550 evaluations have been done to construct the surrogate model. To obtain the surface response of the reference presented in Figs. 6a, c and 7a, c, $26 \times 51 \times 1000=1326000$ model evaluations have been performed. Thus, using this meta-model has divided the computational cost by 520 . Considering the computational time, realizing 1000 simulations with the full model takes $30.2528 \mathrm{~s}$, and so the evaluation of the $26 \times 51$ grid takes $4.0115 \times 10^{4} \mathrm{~s}=11.14 \mathrm{~h}$. Once the PCE-kriging meta-model is constructed, the construction of the surface response for the same grid takes $0.7770 \mathrm{~s}$ as no LHS is 

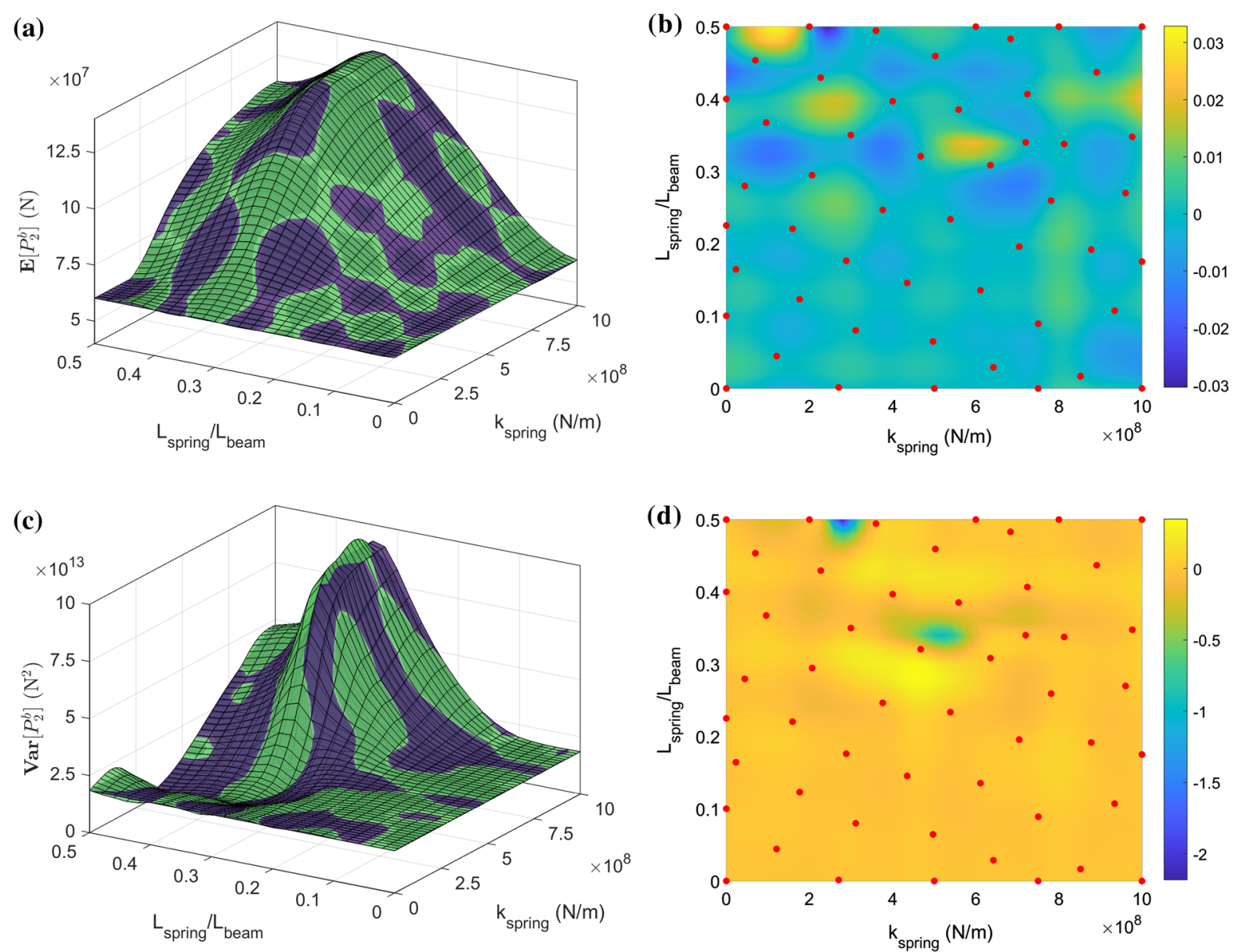

Fig. 7 Buckling load $P_{2}^{b}$-Evolution of the mean (a) and variance (c) for the reference (purple) and PCE-kriging prediction (green) Relative error of the mean (b) and variance $(\mathbf{d})$. Color figure online

required with the formulation. The computational time is then divided here by $5.1628 \times 10^{4}$. The computational interest of using such approach is clearly demonstrated here, even on a small model whose evaluation time is small. This is mainly due to the fact that all the stochastic information is retained in the PCE coefficients parameterized with the kriging, avoiding the realization of costly MCS or LHS to get the stochastic moments.

\subsection{Sensitivity analysis}

To go further in the analysis, the Sobol indices are directly extracted from the PCE coefficients, they are given in Fig. 8 for both buckling loads, and the name of each variable is given at the top of each sub-figure. Only the first-order Sobol indices are displayed here as the total order Sobol indices appear to be equal. This demonstrates the low coupling between the different parameters, and justify the use of a hyperbolic norm as a truncation scheme for the PCE here. As a complement, in Table 5 the minimum and maximum values observed on the grid for each first-order Sobol index are given for each buckling load. The Sobol indices vary a lot from one parameter to another and depends on the considered buckling load. As a reminder, when the Sobol index of a parameter $i$ is close to 1 , then this parameter has a large influence on the output variance, whereas if the Sobol index is near 0, then this parameter $i$ has a low influence. Sobol indices are a convenient tool to evaluate and compare the influence of different parameters on a quantity of interest. 



Fig. 8 First-order Sobol indices for the first (a) and second (b) buckling loads 
Table 5 Minimum and maximum values over the $\left(k_{\text {spring }}, L_{\text {spring }}\right)$ grid of first-order Sobol indices for each variable and for each buckling load

\begin{tabular}{lllllll}
\hline & $E$ & $L_{\text {beam }}$ & $b_{\mathrm{o}}$ & $\mathrm{h}_{\mathrm{o}}$ & $b_{\mathrm{i}}$ & $h_{\mathrm{i}}$ \\
\hline$S_{i}\left(P_{1}\right)$ & {$[0.0350 ; 0.0708]$} & {$[0.0011 ; 0.2282]$} & {$[0.0710 ; 0.0956]$} & {$[0.6370 ; 0.8496]$} & {$[4.43 \mathrm{e}-4 ; 0.0026]$} & {$[0.0053 ; 0.0096]$} \\
$S_{i}\left(P_{2}\right)$ & {$[0.0517 ; 0.0698]$} & {$[0.0092 ; 0.2437]$} & {$[0.0687 ; 0.0942]$} & {$[0.6214 ; 0.8217]$} & {$[2.21 \mathrm{e}-4 ; 0.0023]$} & {$[0.0066 ; 0.0093]$} \\
\hline
\end{tabular}

Considering the first buckling load, the outer height $h_{o}$ has the highest influence as its Sobol index remains always constant near 0.7. A jump to 0.85 around $L_{\text {spring }} / L_{\text {beam }}=0.5$ and $k_{\text {spring }}$ is however observed, but it corresponds to the area where the error of the meta-model is the highest and so this higher value has to be taken with attention. The second parameter that has the highest influence is the beam length with a Sobol index equal to 0.2, which is much lower than the outer height of the beam. The other parameters have low Sobol indices values, showing their low influence on the first buckling load. Thus, if one wants to reduce the variance on the buckling load, it has to control the uncertainty on the beam length and its outer height in priority.

Considering the second buckling load, similar analysis can be done. The parameter with the highest influence is the outer height $h_{o}$ with a Sobol index of about 0.7 . The latter increases up to 0.8 when the buckling load reaches its threshold value. The length of the beam is the second parameter that influences the second buckling load, with a lower influence as the Sobol index is only equal to about 0.2. The latter drops to about 0.02 , having an evolution complementary to the Sobol index of $h_{o}$.

Sobol indices are usually used to compare and assess the influence of each parameter on the output variance. By ranking the influence of the random parameters on the buckling loads here, the following conclusion is drawn:

- high influence: it corresponds to the parameters with the highest Sobol indices, here $h_{o}$ as the Sobol indices are always superior to 0.62 for both buckling loads.

- medium influence: $L_{\text {beam }}$ can also be considered as moderately influential as the Sobol indices can reach up to 0.2 in some cases. However, $L_{\text {beam }}$ reaches low values in some cases (see minimum equal to 0.0092 and 0.0011 ) so its influence depends on the spring location and stiffness.

- low influence: all the other parameters, namely $E, b_{o}, b_{i}$ and $h_{i}$, have a low influence as their Sobol indices are always inferior to 0.1 and much lower than the other Sobol indices. Their effect can even be neglected based on this analysis.

\section{Conclusion}

In this work, the efficiency of an advanced computational technique based on kriging and Polynomial Chaos Expansion for structural stability of mechanical systems with uncertainties is illustrated. This methodology is more specifically tested in order to predict the buckling behaviour of a beam subjected to random geometrical and material properties and to a deterministic axial compression. In addition, the system includes a punctual spring whose location and stiffness vary, which drastically changes the values of buckling loads. The use of a surrogate model allows reducing the computational burden and avoid a computationally expensive MCS/scanning method. It is demonstrated that the method predicts accurately the buckling loads and reduces drastically the number of model evaluations and the computational time. Finally, it is also illustrated that a sensitivity analysis, without additional computational cost, can be performed to rank the influence of different random parameters.

Acknowledgements J.-J. Sinou acknowledges the support of the Institut Universitaire de France.

Open Access This article is licensed under a Creative Commons Attribution 4.0 International License, which permits use, sharing, adaptation, distribution and reproduction in any medium or format, as long as you give appropriate credit to the original author(s) and the source, provide a link to the Creative Commons licence, and indicate if changes were made. The images or other third party 
material in this article are included in the article's Creative Commons licence, unless indicated otherwise in a credit line to the material. If material is not included in the article's Creative Commons licence and your intended use is not permitted by statutory regulation or exceeds the permitted use, you will need to obtain permission directly from the copyright holder. To view a copy of this licence, visit http://creativecommons.org/licenses/by/4.0/.

\section{References}

1. Jerath S (2020) Structural stability theory and practice: buckling of columns, beams, plates, and shells, vol 184. Wiley, pp 1-672

2. Boyce WE (1961) Buckling of a column with random initial displacements. J Aerosp Sci 28:308-320

3. Lindberg HE (1965) Impact buckling of a thin bar. J Appl Mech 32(2):315-322

4. Fraser WB, Budiansky B (1969) The buckling of a column with random, initial deflections. J Appl Mech 36:233-240

5. Amazigo JC (1971) Buckling of stochastically imperfect columns on nonlinear elastic foundations. Q Appl Math 29:403-410

6. Van Der Neut A (1973) The sensitivity of thin-walled compression members to column axis imperfection. Int J Solids Struct 9(8):999-1011

7. Elishakoff I (1978) Axial impact buckling of a column with random initial imperfections. J Appl Mech 45(2):361-365

8. Elishakoff I (1978) Impact buckling of thin bar via Monte Carlo method. J Appl Mech 45(2):586-590

9. Elishakoff I (1979) Buckling of a stochastically imperfect finite column on a nonlinear elastic foundation: a reliability study. J Appl Mech 46(2):411-416

10. Miller RK, Hedgepeth JM (1979) The buckling of lattice columns with stochastic imperfections. Int J Solids Struct 15(1):73-84

11. Wang X, Elishakoff I, Qiu Z, Ma L (2009) Comparisons of probabilistic and two nonprobabilistic methods for uncertain imperfection sensitivity of a column on a nonlinear mixed quadratic-cubic foundation. J Appl Mech 76(1):011007

12. Verhaeghe W, Elishakoff I, Desmet W, Vandepitte D, Moens D (2013) Uncertain initial imperfections via probabilistic and convex modeling: axial impact buckling of a clamped beam. Comput Struct 121:1-9

13. Lv Z, Liu H (2018) Uncertainty modeling for vibration and buckling behaviors of functionally graded nanobeams in thermal environment. Compos Struct 184:1165-1176

14. Luo Y, Zhan J, Liu P (2019) Buckling assessment of thin-walled plates with uncertain geometrical imperfections based on nonprobabilistic field model. Thin-Walled Struct 145:106435

15. Kleijnen J (2009) Kriging metamodeling in simulation: a review. Eur J Oper Res 192(3):707-716

16. Jones D, Schonlau M, Welch W (1998) Efficient global optimization of expensive black-box functions. Technical report

17. Cortes C, Vapnik V (1995) Support-vector networks. Mach Learn 20(3):273-297

18. McKay M, Beckman R, Conover W (2000) A comparison of three methods for selecting values of input variables in the analysis of output from a computer code. Technometrics 42(1):55-61

19. Wiener N (1938) The homogeneous chaos. Am J Math 60(4):897-936

20. Xiu D, Karniadakis G (2002) The Wiener-Askey polynomial chaos for stochastic differential equations. Technical Report 2

21. Ghanem R, Spanos P (1991) Stochastic finite element method: response statistics. In Stochastic finite elements: a spectral approach. Springer, pp 101-119

22. Zadeh L (1996) Fuzzy sets. In: Zadeh LA (ed) Fuzzy sets, fuzzy logic, and fuzzy systems: selected papers. World Scientific, pp 394-432

23. Adhikari S (2007) Uncertainty propagation in linear systems: an exact solution using random matrix theory. In: 48th AIAA/ASME/ASCE/AHS/ASC structures, structural dynamics, and materials conference, p 1957

24. Falsone G, Settineri D (2013) Explicit solutions for the response probability density function of linear systems subjected to random static loads. Probab Eng Mech 33:86-94

25. Li G, Lu Z, Li L, Ren B (2016) Aleatory and epistemic uncertainties analysis based on non-probabilistic reliability and its kriging solution. Appl Math Model 40(9-10):5703-5716

26. Wu D, Gao W, Song C, Tangaramvong S (2016) Probabilistic interval stability assessment for structures with mixed uncertainty. Struct Saf 58:105-118

27. Feng J, Wu D, Gao W, Li G (2017) Uncertainty analysis for structures with hybrid random and interval parameters using mathematical programming approach. Appl Math Model 48:208-232

28. Denimal E, Nechak L, Sinou J-J, Nacivet S (2018) A novel hybrid surrogate model and its application on a mechanical system subjected to friction-induced vibration. J Sound Vib 434:456-474

29. Lophaven S, Nielsen H, Søndergaard J (2002) DACE: a Matlab kriging toolbox, vol 2. Citeseer

30. Rasmussen C, Williams C (2006) Gaussian processes for machine learning. MIT Press, Cambridge

31. Xiu D, Karniadakis G (2003) Modeling uncertainty in flow simulations via generalized polynomial chaos. J Comput Phys 187(1):137-167

32. Sudret B (2008) Global sensitivity analysis using polynomial chaos expansions. Reliab Eng Syst Saf 93(7):964-979

33. Lüthen N, Marelli S, Sudret B (2020) Sparse polynomial chaos expansions: solvers. Basis adaptivity and meta-selection, Technical report

34. Blatman G, Sudret B (2011) Adaptive sparse polynomial chaos expansion based on least angle regression. J Comput Phys 230(6):2345-2367 
35. Sobol IM, Kucherenko SS (2005) Global sensitivity indices for nonlinear mathematical models. Review. Wilmott 2005(1):56-61

36. Yajuvindra K (2018) The Rayleigh-Ritz method for linear dynamic, static and buckling behavior of beams, shells and plates: A literature review. J Vib Control 24(7):1205-1227

Publisher's Note Springer Nature remains neutral with regard to jurisdictional claims in published maps and institutional affiliations. 